$$
\text { ffor }
$$

Cuadernos de $\mathrm{H}$ ideas

ISSN: 2313-9048

cuadernosdehideas@perio.unlp.edu.ar

Universidad Nacional de La Plata

Argentina

\title{
Convergencia tecnológica, concentración de Medios y pluralidad
}

\author{
Ponza, Pablo \\ Convergencia tecnológica, concentración de Medios y pluralidad \\ Cuadernos de H ideas, vol. 13, núm. 13, 2019 \\ Universidad Nacional de La Plata, Argentina \\ DOI: https://doi.org/10.24215/23139048e025
}

Esta obra está bajo una Licencia Creative Commons Atribución-NoComercial-Compartirlgual 4.0 Internacional. 
Artículos

\section{Convergencia tecnológica, concentración de Medios y pluralidad}

Technological convergence, media concentration and plurality

Pablo Ponza

DOI: https://doi.org/10.24215/23139048e025

Universidad Nacional de Córdoba, Argentina

pabloponza@yahoo.es

(D) http://orcid.org/0000-0001-8421-3940

Recepción: 20 Junio 2019

Aprobación: 11 Noviembre 2019

Publicación: 20 Diciembre 2019

Recepción: 20 Junio 2019

Aprobación: 11 Noviembre 2019

Publicación: 20 Diciembre 2019

\section{RESUMEN:}

Este artículo se propone, como objetivo general, un acercamiento al complejo e imbricado vínculo entre tecnología y medios de comunicación, a partir de la articulación productiva de algunos conceptos clásicos de las Ciencias Sociales. Y, en específico, busca analizar los modos de producción, funcionamiento y desempeño de los medios de comunicación desde una perspectiva crítica que combina aportes de la sociológica, la semiótica y la comunicación. El trabajo se divide en tres partes. La primera analiza esquemáticamente el dominio material que ejercen los soportes tecnológicos sobre el proceso productivo de los medios. El segundo apartado analiza el proceso de convergencia tecnológica y algunos resultados de la fuerte concentración de medios que se registra en Argentina. Y, la tercera, analiza las funciones y mecanismos de control productivo, internalización y conformidad que generan los medios; refiriéndose, diagonalmente, a los efectos cognitivos y narrativos que provoca una hiper-mediación cada vez más dependiente de parámetros tecnológicos.

Palabras Clave: Convergencia tecnológica, Concentración de Medios, Pluralidad.

\section{Abstract:}

This article proposes, as a general objective, an approach to the complex link between technology and media, based on the articulation of some classical Social Sciences concepts. Specifically, the text analyze the diferents ways of production, operation and performance of the medias from a critical perspective, using contributions from sociology, semiotics and communication. The work is divided in three parts. The first one analyzes schematically the material domain that technological media exerts on the media production process. The second section analyzes the process of technological convergence and some results of the strong concentration of medias in Argentina. And, the last one, analyzes the mechanisms of productive control, internalization and conformity generated by the medias; referred to the potencials effects that technology parameters cause on our percepctions and narratives.

KEYWORDS: Technological convergence, Media concentration, Plurality.

\section{INTRODUCCIÓN}

En los últimos años, a propósito de mi labor como docente, investigador y director de diversas tesis de grado y posgrado, he detectado un creciente interés por analizar dos cuestiones que se tornan recurrentes. Por un lado, cuál es el impacto que tienen los discursos y representaciones de los medios de comunicación en la construcción de nuestra identidad e ideología. Por otro, cómo se han transformado los medios en base a las llamadas nuevas tecnologías. Asimismo, no parece casual que esta preocupación se torne recurrente durante los períodos electorales y pre-electorales, es decir, durante aquellos lapsos de tiempo en que los medios de comunicación enfocan plenamente sus recursos y repertorios de acción (Tilly, 2005) en una lucha explícita por lograr la primacía hegemónica del bloque de poder al que representan. 
De esa cíclica preocupación se desprenden diversos interrogantes, algunos de ellos enfocados en el ámbito de la recepción y apropiación de las audiencias y, otros, dirigidos más bien al universo de la producción de los medios.Centrando nuestro análisis en el universo de la producción, son cada vez más frecuentes las preguntas que advierten no sólo una articulación, sino una mixtura entre los llamados medios tradicionales (Lacunza, 2016) y las nuevas tecnologías, por ejemplo:¿qué es la convergencia tecnológica y cómo afecta el mapa de medios? ¿la composición económico-financiera incide en el perfil periodístico y la línea editorial de los medios? ¿cómo impactan los soportes tecnológicos en sus modos de producción?̨cuál es el impacto de las nuevas tecnologías y las redes sociales en nuestras prácticas informativas, en nuestra identidad y nuestra composición ideológica? ¿la polarización es consecuencia de la concentración de medios? ¿es posible una mayor pluralidad de voces en un mapa de medios eminentemente concentrados?

Con la idea de reflexionar alrededor de algunas de las derivas que abren estas preguntas, el texto se propone dos objetivos, uno general y otro específico. El general consiste en realizar un acercamiento al complejo e imbricado vínculo entre tecnología y medios de comunicación, a partir de la articulación productiva de algunos conceptos clásicos de las Ciencias Sociales. Y el específico busca analizar los modos de producción, el funcionamiento y desempeño de los medios de comunicación desde una perspectiva crítica que combine aportes de la sociológica, la semiótica y la comunicación.

Para ello, el trabajo se divide en tres partes. La primera analiza esquemáticamente el dominio material que ejercen los soportes tecnológicos sobre el proceso productivo-comunicacional de los medios. El segundo apartado analiza el proceso de convergencia tecnológica y algunos resultados de la fuerte concentración de medios que se registra en Argentina. Y, la tercera parte, analiza las funciones y mecanismos de control productivo, internalización y conformidad que generan los medios; refiriéndose, diagonalmente, a los efectos cognitivos y narrativos que provocan una hiper-mediación creciente y cada vez más dependiente de parámetros tecnológicos.

Como hipótesis o guía de lectura, el texto propone, por un lado, que la morfología funcional y material de los soportes tecnológicos determinan la super-estructura comunicacional de los medios, e incide, no sólo en sus modos de producción, sino también en la configuración ideológica de su fuerza de trabajo. Y, por otro, consigna que un escenario caracterizado por la concentración en la propiedad de los medios conduce a la polarización, un reduccionismo en las narrativas y una escasa pluralidad de voces en la generación de contenidos.

\section{TECNOLOGÍA, MEDIOS DE PRODUCCIÓN Y MEDIOS DE COMUNICACIÓN}

Sin deseos ni necesidad de exponer un exhaustivo relato delas críticas y aportes que se han realizado desde el encuadre marxista clásico a nuestra área de conocimiento, consideramos de utilidad explicar la lógica de funcionamiento general de los medios de comunicación tradicionales, a través de la metáfora que utilizó Karl Marx (1969) para describir el funcionamiento general del todo social. Es decir, desde una perspectiva materialista y dialéctica extremadamente esquemática -y con fines exclusivamente expositivos-, es posible concebir a los medios de comunicación como un edificio que se construye en dos niveles, uno que funciona como estructura y otro como superestructura. El primer nivel está constituido por una base económicofinanciera, una infraestructura y un soporte tecnológico que hace de cimiento fundante sobre el cual se monta, con posterioridad, el segundo nivel.

El segundo nivel del edificio comunicacional está compuesto por dos instancias: la jurídico-política y la ideología. La instancia jurídico-política consiste en la organización administrativa, las normativas, leyes y formas político-institucionales que controlan la actividad productiva de los medios. Y la ideología, consiste en el sistema de ideas dominantes cuyas nociones ético-morales orientan el sentido de las representaciones y los discursos de los medios. Esto es, la red de valores o todo aquello que se considera correcto, aceptable o conveniente según cual sea la orientación económico-financiera que caracteriza a la estructura. Para Teun 
Van Dijk (2012), las ideologías son aquellas representaciones sociales que definen la identidad social de un grupo o estamento, son creencias compartidas sobre las condiciones fundamentales de un modo de existencia y reproducción. Se trata de creencias axiomáticas compuestas por valores culturales y un conjunto de categorías, actividades y normas morales sobre las que se constituye el sentido de pertenencia y el nosotros como identidad; un nosotros cuya auto-representación positiva implica en sí misma una operación de polarización en cuyo extremo binario se ubica la expresión negativa del otro.

En otras palabras, los medios de comunicación, como cualquier otra empresa con fines de lucro que se desempeña dentro del sistema capitalista actual, se rigen por una lógica financiero-contable de amortizacióninversión que no escapa a las leyes productivas y concéntricas del mercado. Y su funcionamiento se organiza a partir del alineamiento jerarquizado de dos componentes básicos. En primer lugar, los soportes tecnológicos. Y, en segundo término, los procesos de codificación, acceso e intercambio de datos, información o mensajes; cuyo insumo esencial son los signos y significantes del lenguaje.

Por eso, como primera definición, asumimos que los soportes tecnológicos y su morfología funcional constituyen el primer anillo material de dominio de los medios. Un dominio a través del cual, y en un nivel super-estructural, orienta luego todo el proceso de producción comunicacional. De modo que lo que acontece en la base económica y técnica de los medios determina la orientación que luego asume su producción en cuanto a toda forma de representación discursiva. Si bien esta es una descripción elemental que se centra únicamente en aspectos productivos y no en los procesos de recepción o apropiación de las audiencias, nos permite, por un lado, distinguir cuales son los componentes constitutivos de los medios de comunicación y, por otro, advertir como Louis Althusser (1999), que las condiciones materiales de producción de una empresa no pueden ser analizadas ni comprendidas atomizadamente, pues toda empresa se halla inserta en un sistema globalmente integrado, mediado por una circulación de capitales y relaciones de interés sin término.

Si bien el paulatino proceso de concentración en la propiedad de los medios es materia de análisis del segundo apartado de este texto, a modo de avance podemos decir que actualmente hay una convergencia tecnológica en concurrencia con la propiedad de los medios de comunicación que se observa a escala planetaria. Es decir, hay una tendencia mundial que apunta a conjugar sinérgicamente tanto los requerimientos productivos y tecnológicos de las industrias del rubro, como a promover la flexibilidad de los límites jurídicos y políticos de aquellos Estados que obstaculicen su libre expansión. Dicha concentración sería resultado, tanto de una convergencia de soportes, como de un afán por reducir costos devenidos dela concurrencia comercial. Si bien no vamos a profundizar aquí en este aspecto, consideramos oportuno adelantar que esta es la dimensión clave donde se cursan, tanto el dominio material, como la inclinación ideológica de los medios de comunicación.

Por eso, como segunda definición, consignamos que las condiciones de producción y reproducción de cualquier empresa de medios sintetiza en su infraestructura, en todos sus soportes y dispositivos tecnológicos, el resultado de un largo y complejo entramado de relaciones de interés económico y técnico históricamente constituido, cuya trayectoria puede ser desentrañada -paso a paso-por estudios específicos interesados en explicar las razones de su predominio o supremacía. Justamente en dicho entramado de relaciones económicas y técnicas descansaría, para Andrew Feenberg (2005), la relevancia práctica de la corporeidad que precede la construcción de toda comunicación mediatizada.

De modo que, así como la dimensión técnico-productiva determina el sistema de codificación de mensajes de los medios de comunicación, surge coherente pensar que dicho sistema de codificación influye también en planos subjetivos y abstractos como el lenguaje, las narrativas, las construcciones de sentido, los enfoques periodísticos y las líneas editoriales. Y en este punto no hay divergencias, puesto que tanto el papel sobre el que se imprimen los periódicos - para el caso de los medios tradicionales- como la fibra óptica por donde viajan los datos de internet - para el caso de sus expresiones trans-mediales-, están signadas por la misma racionalidad que impone una materialidad dominada por el interés y los principios de su filosofía técnica, cuyo universo 
conceptual está -ante todo- abocado a garantizar la reproducción de las condiciones materiales que permitan su propia sustentabilidad.

En este sentido, ya Marshall McLuhan en 1964 advirtió que las tecnologías son extensiones alienadas en sus creadores. Para McLuhan el enfoque aplicado a las tecnologías dominan nuestra creatividad y restringen el espectro de nuestras percepciones. De allí que no sea posible distinguir entre emisor y mensaje, y por lo tanto no deberíamos considerarlos fenómenos separados sino caras de una misma moneda. El medio es el mensaje y los mensajes representan al medio. Lo mismo ocurre con las llamadas nuevas tecnologías. Así como los medios son el mensaje, la permanente modernización y creación de soportes tecnológicos está abocada a explorar nuevos territorios cognitivos en el foro intelectual y sensitivo de los individuos, puesto que, en la atomizada, confortable y plácida intimidad de los recintos privados, la acción colonizadora de las tecnologías es más sutil y penetrante. Aplicada a los medios, sean los más tradicionales o los más modernos, las tecnologías mutan y se retroalimentan en una transformación cíclica que amplía, pero, a su vez, amputa y controla las opciones de nuestros sentidos.

Según Alvin Tofler (1979), desde hace años la tendencia tecnológica de los medios de comunicación se inclina a desmasificar sus redes y dispositivos de contacto para crear segmentos diferenciados de públicos cada vez más identificados y discriminados en cuanto a prácticas, preferencias y consumos. El interés principal de los desarrolladores de tecnologías para medios estaría enfocado en acceder a la vida íntima de los sujetos mediante canales y plataformas cada vez más personalizadas, en virtud de conseguir la mayor cuota de atención posible para saturarlos de información y estímulos específicos. Para Tofler, el paradigma de la información y el conocimiento en el que nos encontramos actualmente consigna como característica principal la alta velocidad en la producción y circulación de datos interactivos, globalizados, colaborativos, dinámicos, multicausales y caóticos. Este nuevo espacio industrial global se construye en una discontinuidad geográfica donde la producción de datos e informaciones es cada vez más inmediata y descentralizada. Asimismo, dichos datos e informaciones de circulación pública están revestidas por otros estímulos y funciones comunicacionales que no sólo tienden a desarticular la circunscripción espacial y los horarios de los actores, sino también a poner en cuestión la educación y las formas que adoptan nuestras decisiones y nuestras relaciones interpersonales, que son cada vez más remotas, distantes y mediatizadas.

En opinión de Pablo González Casanova (2004), la interpretación dominante de este paradigma del conocimiento, la información y las nuevas tecnologías, es totalmente funcional a un sistema que busca imponer el dominio del mercado como si se tratara de una opción racional e impersonal de interés universal. Sin embargo, no se trata de un interés universal e impersonal sino de intereses particulares que benefician especialmente a pequeñas minorías altamente concentradas. Sin una perspectiva crítica de este paradigma de la información, el conocimiento y las nuevas tecnologías, contribuiríamos solamente a perfeccionar la dominación y la explotación de los poderosos, y no a la equidad entre humanos. En ese contexto, la información y el conocimiento sólo parecen ser asimilables por nuestra experiencia en la medida que sean de utilidad para el sistema tecnológico. De allí que la tecnología circule y se negocie como cualquier otra mercancía, cuyo valor se establece en directa relación con los proyectos dominantes.

Para Herbert Marcuse (1998), la dominación tecnológica -que también se expresa en los medios de comunicación- es una más de las diferencias materiales que impone una institucionalización técnicamente mediadas por relaciones de dominación. Pero configurar la tecnología y los medios de comunicación para reproducir el dominio de unos sobre otros no es la única posibilidad de esa acción técnica. Según Feenberg (2005), la orientación y el ordenamiento actual de las tecnologías no es algo dado e inmutable, pues la tecnología dispone de potencialidades benéficas y humanizantes que han sido cooptadas por un orden que controla y restringe la participación en su diseño y desarrollo a pequeños cenáculos de poder que excluye a las mayorías.

Por último, y volviendo a nuestra descripción inicial, cabe preguntarse ¿cuál es el lugar que ocupa la fuerza de trabajo en el edificio comunicacional? Desde la perspectiva de los dueños del capital y los 
núcleos de decisión tecnocrática, la mano de obra ocupa un rol subalterno y de gran fragilidad. Y esto se comprueba de dos modos. Por un lado, objetivamente, cotejando la inestable precariedad contractual en la que mayoritariamente se desempeñan sus trabajadores. Y, por otro, subjetivamente, a través de la opinión y la conducta de los propios técnicos, ingenieros, diseñadores, gestores de comunidades, redactores, periodistas o directores de sección que integran el sistema productivo de los medios, quienes -sin importar la tarea que realicen- reconocen mansamente que son prescindibles, que son recambiables, y que hay un larga fila de jóvenes que esperan ansiosos ocupar sus puestos, no sólo a un menor costo manteniendo la calificación y el rendimiento respecto de las exigencias impuestas por la división técnico-productiva actual, sino, además, con un apego sin fisuras al perfil ideológico de sus empleadores.

Si bien analizar nuestra cultura científica y nuestro sistema educacional no es parte de los objetivos propuestos por este texto, sin dudas es una de sus derivas. La actual configuración de los medios de comunicación nos conduce a reflexionar sobre su plena subordinación a las necesidades de un capitalismo avanzado que instruye a los sujetos para que sean capaces, no sólo de ocupar eficazmente sus puestos en la esfera productiva, sino también para que sean respetuosos de las reglas, las normas morales y de conciencia profesional establecida por el sistema donde se insertan. En palabras de Althusser (1999: p.107): «la reproducción de la fuerza de trabajo no sólo exige la reproducción de su calificación, sino, al mismo tiempo, la reproducción de la sumisión de los trabajadores a las reglas del orden establecido, es decir, a la reproducción de su sumisión a la ideología dominante». Por eso, para el sentido de supervivencia más ramplón y generalizado de un trabajador medio, es sabido que para desenvolverse correctamente en una empresa, para no temer ser despedido y conservar la fuente laboral de la que depende su subsistencia, no sólo es necesario disponer de la experticia profesional, sino también saber mantener la boca cerrada y los pies en el plato, conocer la agenda que define los temas de debate, saber cuáles son los foros legitimados de intercambio, quiénes son los protagonistas y antagonistas, y sobre todo tener bien claro cuál es el universo de enunciación políticamente correcto dentro del espacio ideológico que se ocupa y al que se representa.

\section{Medios de COMUNiCACión, CONCENTRACión y PLURALIDAD}

Como dijimos, el proceso de concentración en la propiedad de los medios es una tendencia mundial que se ha acentuado en los últimos treinta años, y Argentina se ha convertido en un caso paradigmático de concentración en América Latina. Robert McChesney y John Nichols (2002) nos advierten que la convergencia comercial global en infocomunicaciones que se observa en todo occidente perseguiría dos grandes metas. La primera, conjugar sinérgicamente los requerimientos productivos y tecnológicos de las industrias del rubro. Y, la segunda, promover la flexibilidad de los marcos jurídicos y políticos de aquellos Estados que obstaculicen su libre expansión territorial. Dichas metas devienen de la dinámica acumulativa de los capitales, de su afán de lucro, de su sistemática necesidad de bajar costos impositivos, de mano de obra, de logística, producción, emisión, distribución o reproducción. Pero ¿en qué consiste la convergencia infocomunicacional?

Para Henry Jenkins (2008) la convergencia info-comunicacional consiste en la concurrencia de los soportes y contenidos que fluyen a través de múltiples plataformas mediáticas. Dicha convergencia es producto de la paulatina coexistencia y superposición tecnológica de los soportes y canales de distribución de medios, pero también de un progresivo proceso de transformación cultural basado en prácticas más participativas por parte de los usuarios. Los consumidores buscan nuevas informaciones para establecer conexiones entre contenidos dispersos como una práctica habitual. Sin embargo, la aparente libertad con la que podemos participar de las redes digitales presenta una contradicción insuperable caracterizada por la alarmante concentración de la propiedad de los medios comerciales dominantes, que reúne en un puñado de conglomerados mediáticos multinacionales el dominio de todos los sectores de esta industria. 
En América Latina, el proceso de concentración de medios comenzó con las políticas neo-liberales implementadas en la década de 1990. En ese período, por un lado, comenzaron a retraerse significativamente las capacidades y el arbitrio de los Estados nacionales en el ámbito de la producción y distribución de bienes y servicios culturales. Y, por otro, se favoreció la alianza o cooperación solidaria entre dueños de empresas info-comunicacionales y otros agentes del poder económico con injerencia en temas estratégicos. Desde entonces, según Guillermo Mastrini y Martín Becerra (2009), Argentina, Uruguay, Chile, Brasil, Bolivia, Perú, Colombia, Venezuela y México, han venido consolidando un escenario oligopólico cuyo principal efecto se registra en la fuerte incidencia empresarial en los procesos de construcción de discursos periodísticos.

Según Glenn Postolski, Ana Santucho y Daniel Rodríguez (2003) en Argentina habría tres grupos de medios dominantes. En primer lugar, Clarín y Telefónica, destacados tanto por la cantidad de medios que poseen como por la diversidad de sectores que abarcan. Ambos integran HMT\&F, Cisneros, AT\&T, Liberty Media, UGC, CIE, Recoletos, Prisa o Pearson's, un holding donde capitales extranjeros mayoritariamente de origen norteamericano mantienen relaciones comerciales con otros grupos locales y europeos con quienes comparten la propiedad de distintas empresas del rubro. En segundo lugar, se encuentran TyC-Avila y UnoVila de menor envergadura y complejidad. Y, en tercer lugar, un conjunto de empresas como La Nación y Editorial Perfil cuya presencia en el periodismo gráfico es altamente considerable.

Sin dudas el caso de Clarín, tras la fusión realizada en 2017 entre Telecom y Cablevisión, ha hecho realidad la mayor concentración en info-comunicaciones de la historia argentina y de todo América Latina. De este modo, según María Gabriela Ensinck (2019), Telecom-Cablevisión se ha convertido en la tercera empresa con mayor facturación del país tras Mercado Libre e YPF. En la relación accionaria, David Martínez, dueño de Fintech, es el principal socio con el $41 \%$ de las acciones. Por su parte, Cablevisión Holding (propiedad del Grupo Clarín) continuará en manos de sus tradicionales dueños, que retienen un 33\% del paquete accionario. Según Media Ownership Monitor Argentina (2019) en un informe publicado por Reporteros Sin Fronteras, solamente cinco accionistas concentran el 71\% de las acciones del Grupo Clarín, 29,8\% Héctor Magneto, 24,8\% Marcela Noble Herrera, 24,8\%, Felipe Noble Herrera, 10,3\% José Aranda y 10,2\% Lucio Pagliaro. Además, un $9 \%$ es propiedad de GS Unidos LLC y la Compañía norteamericana Ralph Booth; mientras que el 20\% restante cotiza en las bolsas de comercio de Buenos Aires y Londres.

Según Pablo Fernández Blanco (2018), Telecom dispone en Argentina de la impresionante cifra de 18,98 millones de clientes y 1,75 millones de suscriptores de Arnet, con ventas por 65.186 millones de pesos y ganancias de 7.724 millones de pesos. Por su parte, Cablevisión S.A. tiene 2,3 millones de clientes de banda ancha de Fibertel y 3,5 millones de televisión por cable, consignando ventas por 40.952 millones de pesos, con ganancias de 5.895 millones de pesos. Para Patricia Valli (2019), como resultado de dicha fusión, esta nueva megaempresa controla el 57\% del mercado de banda ancha del país, que, en cinco años, le reportará ganancias de alrededor de 1.700 millones de pesos, ahorrando entre un 1,1\% y 1,5\% anual por la sinergia alcanzada por la unión de las dos firmas. Para Fernández Blanco (2018) del diario La Nación, un inversor que tenía acciones en el grupo Clarín en enero de 2012 en plena pelea con el gobierno de Cristina Fernández de Kirchner (2008-2015), ha obtenido a la fecha una ganancia del 3.074\%.

Según publica el Boletín Oficial de la República Argentina, el Ente Nacional de Comunicaciones (ENACOM ex AFCA) dirigido por Esteban Greco, habilitó a Telecom Argentina S.A. a absorber a Cablevisión S.A. mediante la Resolución 5644-E/2017 del 21/12/2017, a propósito de las actuaciones iniciadas el 29 de diciembre de 2015 por Telecom Argentina S.A. y Cablevisión S.A. -expediente EX-2017-20912532-APN-AMEYS\#, la Ley $N^{\circ} 27.078$, la Ley $N^{\circ} 26.522$, y el Decreto $N^{\circ} 267-$, donde consta que esta fusión fue resultado de las actuaciones realizada por las propias firmas, que, según las normas mencionadas, se establece su habilitación para brindar servicios que comprenden, además de televisión por cable, los servicios de Telefonía Móvil (STM), de Radiocomunicaciones Móvil Celular (SRMC), Comunicaciones Personales (PCS) y Comunicaciones Móviles Avanzadas (SCMA), así como sus futuras evoluciones tecnológicas. Esto significa que Telecom-Cablevisión puede comercializar el llamado cuádruple 
play, es decir, diversas opciones de servicios paquetizados que combinan internet, telefonía fija, celular y televisión a través de un mismo soporte.

Que el ENACOM haya favorecido la vocación concentradora de estas compañías permitiéndoles conjugar sinérgicamente sus intereses financieros con sus requerimientos tecnológicos, da lugar a los argumentos de Gabriel Sosa Plata (2016), quien señala que la concentración de medios siempre ha sido más sencilla en contextos regionales de alta debilidad institucional, escasa politización social y baja complejidad económica. Contrariamente, esta clase de capitales han encontrado mayores dificultades para su expansión oligopólica o monopólica en aquellos países donde la legislación fomenta una competencia real y favorece el acceso a la comunicación concibiéndola como un derecho humano, y no como un bien económico sujeto a las reglas del mercado.

En el caso argentino, el ENACOM lejos de favorecer la competencia priorizó los intereses empresariales de Telecom y Cablevisión, flexibilizando los marcos regulatorios que obstaculizaban su libre expansión territorial permitiéndoles consolidar aún más su posición de privilegio. La fusión Telecom-Cablevisión es la mayor concentración de infraestructuras en telecomunicaciones de nuestro país, que es la clave material de la primacía hegemónica, puesto que quien domina el tendido de fibra óptica domina también el soporte a través del cual viajan los datos de internet y del llamado cuádruple play, capturando la mayor oferta de servicios, de prestadores y de las opciones de consumo para los usuarios.

Como señalamos al principio, hay dos elementos que nos interesa destacar de este proceso de convergencia y concentración. En primer término, la dimensión material como clave dominante de toda inclinación ideológica de los medios. Y, en segundo lugar, consignar que las condiciones de producción y reproducción de cualquier empresa de medios sintetiza en su infraestructura, en sus soportes y dispositivos tecnológicos, el resultado de un largo y complejo entramado de relaciones de interés económico y técnico que ha sido constituido históricamente, y cuya trayectoria puede ser desentrañada por estudios específicos.

En este sentido, no es la primera vez que el Grupo Clarín no encuentra límites para beneficiarse de su posición dominante y apropiarse de los soportes materiales fundamentales de su rubro para imponer condiciones comerciales. Recordemos, por ejemplo, el caso de Papel Prensa, operada desde 1976 por un directorio de accionistas donde el Grupo Clarín se quedó con el $49 \%$ de las acciones, mientras que La Nación con el 22,49\%, el Estado Nacional con el 27,46\% y la agencia oficial de noticias TELAM con el $0,62 \%$. Los diarios eran en aquel entonces uno de los medios de mayor alcance e influencia, motivo por el cual dominar la producción y distribución de papel prensa tenía un valor estratégico. Papel Prensa estuvo envuelta durante décadas en controversias, no sólo por el manejo oligopólico del papel, sino por la supuesta apropiación compulsiva y violenta de la que fue objeto durante la última Dictadura Militar.

Para Celeste Vásquez (2017) durante la última Dictadura, la prensa era un sector estratégico para los militares, centralmente para poder legitimar el golpe y operar un aparato propagandístico que ocultara sus crímenes de Lesa Humanidad. Las sospechas se dirigen a supuestos favores periodísticos -el tratamiento benigno de los grandes diarios capitalinos- a cambio de su participación en el control de la producción y distribución del papel de diario. Dicha participación fue y es hasta la actualidad un diferencial comercial que determinó la supremacía de Clarín y La Nación entre los medios gráficos nacionales, una supremacía que aún hoy continúa, pues La Nación y Clarín siguen siendo socios del Estado en la única empresa que produce pasta de celulosa en este país, un insumo fundamental para fabricar y abastecer a más de 170 diarios.

Otro dato paradójico, en cuanto a temas de infraestructuras y convergencia info-comunicacional, es la política de desarrollo del propio Estado Argentino, que financia buena parte de las costosas obras de tendido de la fibra óptica que será explotada luego por compañías privadas como Telecom-Cablevisión. Concretamente en 2009, durante la presidencia de Cristina Fernández de Kirchner, se lanzó la empresa estatal Arsat, cuyo objetivo era alcanzar un tendido de 58.000 kilómetros de fibra óptica para servicios de telecomunicaciones en todo el país. Según José Crettaz (2017) en junio de 2017 Arsat llegó a los 22.500 kilómetros, es decir, a más del $60 \%$ del tendido previsto. Estas obras, que continúan bajo la 
gestión de Mauricio Macri, son financiadas con una partida presupuestaria de 1.300 millones de pesos provenientes del Fondo de Servicio Universal, que se nutre del 1\% de la facturación bruta de los operadores de telecomunicaciones. Como vemos, en este caso si podemos decir que el dinero de contribuyentes como Telecom-Cablevisión vuelve en obras. Por cierto, en obras realizadas por empresas constructoras directamente ligadas al Estado y el lobby empresarial cercano al gobierno de turno.

Con la producción de contenidos y gestión de programación ocurre lo mismo, pues los grupos concentrados de medios suelen ser accionistas o propietarios de las productoras de TV, radios, diarios y revistas. Controlan también las productoras de cine y espectáculos, agencias de noticias, discográficas, editoriales y portales de Internet, de los cuales a su vez abrevan otros medios. Entonces, por ejemplo, si seguimos un debate político a través de los diarios, la televisión o la radio, advertimos que un medio cita como fuente a otro medio del mismo grupo, que, a su vez, cita a otro en una espiral de incidencias sin término que se retroalimentan a sí mismas en la construcción de sus relatos.

Martín Becerra (2019), en «Concentración mediática, un símbolo de la era Macri» señala que los cuatro principales grupos de medios argentinos acaparan el 74\% de los diarios, y no sólo concentran el 57,7\% de la audiencia en TV abierta, y el 53\% en radio, sino que, como añadido, acaparan más del $60 \%$ de los contenidos que transmiten las emisoras de TV fuera del área metropolitana de Buenos Aires. Dichos contenidos tienen origen en la capital, en particular en las cabeceras de las dos redes más grandes, Telefé (Viacom) y El Trece (Grupo Clarín). Clarín es el número uno en audiencia televisiva con el 22,6\%, seguido por Telefé con el $15,1 \%$. Es decir, en directa relación con la concentración de la propiedad de medios en pocas manos, se produce además una centralización geográfica en la generación de contenidos, afectando la pluralidad y profundizando las hondas asimetrías culturales, políticas, económicas y sociales existentes en nuestro país.

En resumen, los grupos concentrados controlan y operan sobre toda la cadena productiva, desde los soportes tecnológicos, pasando por la oferta de servicios y contenidos, hasta las narrativas, relatos y cadenas de sentido que adoptan los discursos periodísticos. Por eso, sin legislación y controles que eviten los monopolios, la convergencia en info-comunicaciones tiende a la concentración de medios, la concentración de medios conduce a posturas convergentes y monocordes en los grandes debates nuestro tiempo, y las posturas convergentes y monocordes llevan a una escasa pluralidad de voces. A su vez los debates son eminentemente mediatizados, a los cuales las audiencias concurren a través de sus pantallas, generando un círculo vicioso en el que los grupos dominantes imponen las reglas del juego al resto de los actores y captan cada vez más porciones de los ingresos por servicios, publicidad privada y publicidad oficial, resultante del lobby político que les permite su posición de privilegio.

Según un informe de la UNESCO (2014) sobre libertad de expresión y desarrollo de medios en América Latina y el Caribe, la concentración de medios es la principal causa de la uniformidad de contenidos, el control de temas en la agenda informativa y la escasa pluralidad de voces. Ante este panorama, cabe preguntarse si Clarín, o cualquier otro grupo de medios concentrados ¿proyectará a sus audiencias una visión imparcial de la realidad? ¿construirá representaciones y discursos que socaven sus propios intereses? ¿aplicará un recorte y una articulación selectiva de sentidos a sus relatos? o ¿preferirán presentarse como periodismo independiente, objetivo y/o neutral?

Considerar el problema del pluralismo de los medios por dentro de su propio paradigma es como un perro que se muerde la cola. Es decir, ¿qué clase de horizonte plural puede elaborar el discurso de los medios cuando su propia naturaleza concéntrica margina de los debates de interés público a expresiones que representan espacios políticos y socio-culturales diferentes? Este es un dilema difícil de superar, en especial si tenemos en cuenta una tendencia que prevé agudizar la hiper-mediación de las relaciones, las prácticas y los consumos por la vía tecnológica.

Un ejemplo concreto de uniformidad de contenidos, el control de temas en la agenda informativa y la escasa pluralidad de voces en los medios, es la construcción polarizada de campos de pertenencia políticoideológica y la confrontación binaria entre kirchnerismo-anti-kirchnerismo. Durante la última década 
diarios como La Nación y Clarín, pero también Página 12, foguearon un escenario dicotómico a través de representaciones impugnatorias de su adversario. Estos diarios dieron lugar a relatos fragmentarios que fortalecieron identidades políticas mutuamente excluyentes, imprimiendo una suerte de sesgo cognitivo cuya meta era desacreditar compactamente los argumentos de su adversario (Ponza, 2018). Porque lo importante no eran los argumentos, o la verdad, sino quiénes daban esas razones, y si esos argumentos reforzaban o no la propia cadena de sentidos. El objetivo de esta operación era instalar una agenda de debate sesgado, pero con suficiente valor productivo para dar credibilidad a uno de los bloques en disputa, con el fin de establecer una frontera antagónica en la oferta electoral argentina.

Como añadido, a la escasa pluralidad de voces hay que agregar la uniformidad y la polarización que provocan en las prácticas y consumos la actual organización lógica de los algoritmos en redes como Facebook, Twiter o Instagram, que tipifican las interacciones de los usuarios a base de campos de afinidad. Los algoritmos, que no son ni verdaderos ni falsos, son un principio de organización diseñado para vincular personas y contenidos identificando la frecuencia de sus propias interacciones, conduciendo a una paulatina homogeneidad y polarización en los contactos.

Nosotros somos el amor, ellos son el odio. Nosotros somos la verdad, ellos la mentira. Nosotros somos el futuro, ellos el pasado. Es sencillo encontrar expresiones polarizadas y ficticias como éstas en cualquier medio de comunicación durante una campaña política. Expresiones que tipifican el bien y el mal a partir de un lenguaje que divide dicotómicamente y sin complejidades un mundo ideal, un mundo compuesto por extremos que representan dos proyectos pre-hegemónicos en disputa. En un extremo se ubican los amigos y en el otro los enemigos. En una cara de la moneda se acuña el bien y en la otra el mal. De un lado se ubica la promesa y del otro la amenaza.

Este escenario sesgado, este escenario compuesto únicamente por antípodas, no sólo niega la pluralidad y evade la auto-crítica, sino que está destinada a incluir compulsivamente a los indecisos. Pues en esta contienda el dilema es sencillo: ¿estás con nosotros o en nuestra contra? En esa grieta no hay puntos medios, hay que decidirse rápido, no hay tiempo para debatir otras complejidades, de la urgencia depende el éxito. Los adversarios, los diletantes y los críticos se ubican en el extremo opuesto y son responsables por acción u omisión de que nuestra comunidad imaginada, nuestro mundo ideal, no pueda realizarse. Para James Brown (1991: 25) el éxito de esta clase de operaciones responde a que «la mayoría de la gente quiere creer que los problemas son sencillos en vez de complejos, espera que se confirmen sus prejuicios, quiere sentir que no está marginada, lo que implica que los otros sí lo están, y necesitan señalar a un enemigo que cargue con sus frustraciones».

\section{LA CONCENTRACIÓN DE MEDIOS, EL CONTROL PRODUCTIVO Y LA IDENTIFICACIÓN}

Es posible identificar dos grandes efectos o resultados devenidos del proceso de convergencia tecnológica y de concentración técnico-productiva, en especial cuando esta se da en concurrencia con la propiedad de los medios de comunicación. En este apartado nos referiremos, en primer término, a la implantación de rutinas y procedimientos comunicacionales con funciones de estandarización. Y, en segundo lugar, al paulatino proceso de colonización cognitiva con que estas funciones impactan en nuestras subjetividades.

Sin embargo, antes de avanzar queremos indicar que las reflexiones que elaboramos a continuación deben ser interpretadas en teniendo en cuenta las definiciones consignadas en el primer aparado. Esto es, en primer lugar, que los soportes tecnológicos y su morfología funcional constituyen el primer anillo material de dominio de los medios de comunicación. En segundo término, que las condiciones de producción y reproducción de cualquier empresa de medios sintetiza en todos sus soportes y dispositivos tecnológicos el resultado de un largo y complejo entramado de relaciones de interés económico y técnico históricamente constituido. Y, en tercer lugar, que la tendencia actual apunta a conjugar sinérgicamente tanto los requerimientos productivos y tecnológicos de las industrias del rubro, como a promover la flexibilidad de los 
límites jurídicos y políticos de aquellos Estados que obstaculicen su libre expansión... Consideramos que; si la convergencia en el dominio de los soportes tecnológicos se produce en sinérgica concurrencia con el dominio de los medios de comunicación, convierte dicha concentración en una impresionante usina de poder con múltiples capacidades.

Asimismo, cabe aclarar, que cuando nos referimos a las capacidades del poder tecnocrático y de los medios de comunicación, no estamos pensando en abstracto, como se si tratara de poderes sobrenaturales o de fuerzas celestiales, sino todo lo contrario. Pensamos más bien en personas físicas, en empresas, en actores económicos, políticos y técnicos integrados globalmente, como es, por ejemplo, el caso de Clarín en Argentina, cuya capacidad de negociación local -fundamentalmente a través del lobby político y comercialestá lo suficientemente integrado como para establecer una estrategia de acción coordinada de intereses, con independencia del quehacer deliberado (sea individual o colectivo) que encarnan las audiencias en el plano de la recepción.

Centrados ahora en el plano de las subjetividades, es posible identificar dos efectos o resultados devenidos del proceso de convergencia tecnológica y de concentración técnico-productiva, en especial cuando esta se da en concurrencia con la propiedad de los medios de comunicación. Nos referiremos, en primer término, a la implantación de rutinas y procedimientos comunicacionales con funciones de estandarización. Y, en segundo lugar, al paulatino proceso de colonización cognitiva con que estas funciones impactan en nuestras subjetividades, en la construcción de nuestra identidad y nuestra ideología.

En cuanto a la implantación de rutinas y funciones de estandarización, Eduard Ross (1969) ha analizado la idea de orden y control social productivo a través de tres grandes mecanismos que intervienen en el proceso de internalización y conformidad que desde temprana edad modelan los comportamientos de los sujetos. El primero de dichos mecanismos sería el de reacción o respuesta frente a conductas tipificadas por el orden jurídico. El segundo, el control social formal o primario, aplicado por aquellas instituciones que tienen como función la prevención y la represión: cárceles, geriátricos, establecimientos sanitarios, centros de rehabilitación, entre otras. Y el tercero, el control difuso o indirecto, donde la familia, los grupos religiosos y fundamentalmente los medios de comunicación, promueven o cuestionan las conductas y valores estandarizados por el grupo.

Foucault (2014) sostiene que la eficacia del orden y el control disciplinario no proviene, principalmente, de su carácter represivo sino productivo. Es decir, la eficacia del orden y el control provienen de las usinas generadoras de significaciones complejas, multidireccionales, continuas y azarosas que rigen la sociedad y que sutilmente nos abordan mediante la seducción, el hedonismo, la acción de las tecnologías y los medios. En Las palabras y las cosas (1966), en Vigilar y castigar (1975), y, especialmente en La microfísica del poder (1978), Foucault conceptualiza el poder como una microfísica que se ejerce en red y se expresa en las capacidades para definir qué es verdad, qué es correcto, qué es patológico y qué es normal. Por ello, para Foucault poderoso es quien dispone de las capacidades y el saber técnico para imponer las interpretaciones dominantes de la realidad, es decir, para implantar rutinas y procedimientos comunicacionales estandarizados que se expresan, de modo cada vez más significativo, a través de los medios.

Reforzando esta idea, pero desde las antípodas ideológicas de Foucault, Edward Bernays (1995) explica este proceso aplicando una perspectiva mercantilista-no crítica. Se refiere a la estandarización con la idea del gobierno invisible de la elite o ingeniería mediatizada del consenso. Ubicado en un paradigma democrático netamente liberal y tecnocrático, Bernays se jacta de que el liderazgo de pequeños grupos de poder se construye financiando campañas políticas y mediáticas, pues saben que para lograr avanzar en el sentido de sus intereses es imprescindible que el electorado se incline en su favor y dé su conformidad en las urnas votando a los candidatos de su conveniencia. De allí -dice Bernays- surge el papel protagónico de los medios de comunicación que manipulan opiniones con fines propagandísticos en todas sus formas y secciones. Sin embargo, aclara Bernays, la información y las secciones no son importantes en sí mismas. Lo verdaderamente importante -y que no advierte la lectura ingenua del público masivo- es que el objetivo de los medios no 
es informar sino marcar una agenda de debates cuyo valor productivo consiste en construir credibilidad y respeto alrededor de una autoridad legitimada, y un fuerte descrédito del adversario.

Ignacio Ramonet (2013) dice que ya no es posible hablar de medios independientes del poder político o económico, pues la concentración dominante los ha convertido en actores cuya misión central es difundir su interés e ideología, aunque en apariencia se dediquen al entretenimiento o la difusión de información. La interpelación que los medios hacen a la sociedad a través de temas diversos, como el movimiento de tropas en el exterior, las patentes de un laboratorio, o la suba de los valores de la bolsa en Wall Street, buscan dominar de qué hablan las personas, pero especialmente definir quiénes están autorizados para opinar de esos temas en los medios.

Noam Chomsky (2007), señala que la acción de la tecnología, los medios y la propaganda sobre la opinión pública es a la democracia lo que la cachiporra era antiguamente al estado totalitario. La opinión pública es como un rebaño - dice Chomsky- si se desorienta surge la amenaza de la dispersión y las alternativas transformadoras, por ello es preciso direccionar su atención implantando rutinas comunicacionales con funciones de estandarización que constriña a un rebaño que nunca está del todo domesticado. Para Chomsky controlar la opinión pública es una batalla diaria y permanente, orientada a internalizar cierta conformidad respecto de los liderazgos, el orden social, cultural y político, pero, por sobre todas las cosas, dirigida a consolidar las bases fundamentales del orden económico. Algo que se consigue taladrando sin pausa, día y noche, con estímulos multiplataforma que hacen a la construcción de sentidos a través de narraciones fragmentadas e hiper-mediatizadas.

El segundo de los efectos o resultados que favorece el dominio y la orientación actual de la tecnología, en especial cuando están en concurrencia con los medios de comunicación, es el paulatino proceso de colonización y dominio de las subjetividades. Norbert Elias (1994) señala que las personas no somos inmunes a la multiplicidad de estímulos mediáticos que recibimos, e incesantemente estamos sometidos a un proceso de selección, internalización y conformidad que impacta en nuestras percepciones y comportamientos. Dicha internalización redunda en una suerte de auto-coacción o auto-regulación que nos incluye en los marcos identificables de lo normal, lo civilizado, lo esperable. En su forma ideal esa identificación genera un autocontrol constante y moderado que se expresa en manifestaciones emocionales aceptadas que preservan el ordenamiento de las significaciones estandarizadas.

Para Elliot Aronson (2000), dicha identificación, a diferencia de la imitación, implica creer en las opiniones y valores de identificación que un sujeto se inclina a adoptar. Una creencia es una idea o pensamiento que se asume como verdadero, aunque no lo sea. La verdad es relativa, pues todo depende del cristal con que se mire. En este sentido, la creencia es una construcción subjetiva, es más bien una convicción compartida por un grupo de pertenencia que una certeza. ¿Qué lugar tienen los medios de comunicación en la construcción de creencias? ¿qué convicciones revisten sus representaciones? Para Sigmund Freud (2010), las creencias surgen de una identificación y no de la simple imitación. Es decir, las creencias son resultado de la apropiación de una cadena de significantes inconscientes y una serie de razonamientos que no llegan a la conciencia, y que consisten en ubicarse en el lugar del otro a partir del reconocimiento de algo en común, algo que el otro tiene y que yo deseo.

Ejemplifiquemos este proceso en base a un caso ciertamente ajeno a nuestro tiempo, pero que nos permita advertir más claramente esta proyección abstracta. En el libro Los condenados de la tierra Frantz Fanon (1961) describe descarnadamente los más brutales mecanismos de control y dominación colonial utilizados por el ejército de ocupación francesa en Argelia. Si bien Fanon analiza allí los métodos de una experiencia de militarización y violencia extrema, presenta algunos argumentos homologables para ejemplificar el proceso de identificación, conformidad e internalización que hemos expuesto más arriba a través de Elias, Aronson y Freud. Fanon sostiene que el sujeto, una vez colonizado, en realidad pasa a ser un perseguido que sueña convertirse en colonizador. Un colonizado que no sólo desea tener una hacienda y una mujer como la del colonizador, sino que, además, desea ocupar su rol y poseer su prestigio social. En efecto, dice Fanon, 
el dominado quiere ser como el dominador porque se identifica con él, porque su subjetividad ha sido colonizada por sus valores, sus significaciones y sus tecnologías. En resumen, la subjetividad del colonizado está dominada cuando anhela, ambiciona y envidia ser parte de un mundo al que no pertenece, ni al que va a pertenecer nunca, a pesar de todos los sacrificios que esté dispuesto a hacer para lograrlo.

En el prólogo de Los Condenados de la Tierra, Jean Paul Sartre (1961) asegura que el despliegue objetivo de la racionalidad occidental implicó, necesariamente, un proceso de mutación subjetiva en los argelinos, pues el dominio y el control imperial no sólo usó las fuerzas represivas para mantener a raya a los rebeldes, sino que proyectó sobre la población valores particularmente europeos como si fueran valores universales, logrando un adoctrinamiento por la vía de la identificación.

En el plano psicológico, podemos decir que esta clase de identificación funciona como una enajenación estructural que es parte del proceso de definición y conocimiento primitivo del yo. Jacques Lacan (2013) define esa identificación como una transformación que se produce en el sujeto cuando asume una imagen como propia. Incluso distingue entre identificación imaginaria e identificación simbólica, la primera de ellas como parte de la teoría del espejo, es decir, la identificación con el otro por intermedio del deseo, que es una identificación primaria formadora del yo. Y, la segunda, la identificación simbólica que se observa en la etapa adulta, resultante de habitar el lenguaje con los significantes insignia de Otro, o lo que Lacan denomina la omnipotencia del Otro, una identificación que modela el comportamiento humano e integra los sujetos a una determinada cultura. En La psicología de las masas y análisis del yo, Sigmund Freud (2010) señala que toda psicología social es también individual, pues el sujeto siempre toma en cuenta al otro, tanto sea como modelo a seguir e imitar, como objeto de amor, como enemigo, como auxiliar, como amigo o compañero.

Si pensamos por un momento en la concentración de los medios de comunicación y en la transformación de nuestros consumos y prácticas comunicacionales en los últimos quince o veinte años, no es difícil advertir cómo diferentes dispositivos tecnológicos han colonizado un amplio territorio de nuestra vida cotidiana, tanto a nivel material como cognitivo. Dicha transformación implica el despliegue objetivo de una racionalidad y una materialidad que ha implicado, necesariamente, un proceso de mutación subjetiva. Aunque sea cotidiano y sutil, el impacto se hace evidente, por ejemplo, cada mañana cuando nuestra primera acción al levantarnos es encender el teléfono y, por la noche, la última es apagarlo (si es que lo apagamos). Auto-comprobamos así nuestra dócil aceptación a someternos durante todo el día, todo el año, a un frenético, creciente e incesante bombardeo de estímulos trans-mediáticos del que difícilmente podemos evadirnos. Si bien este texto no tiene el propósito de analizar la recepción activa ni la apropiación de las audiencias, es esperable pensar que ese bombardeo no solo incide sobre nuestras relaciones interpersonales, nuestras percepciones y decisiones, sino también en el carácter que adoptan nuestros procesos cognitivos y narrativos cuando vamos a participar o intervenimos en cualquier intercambio o conversación. Cuando nos referimos al impacto que tienen las nuevas tecnologías en nuestros procesos cognitivos y narrativos, estamos pensando en los parámetros tecnológicos para la comunicación, por ejemplo, Instagram acepta videos con una duración máxima de cincuenta y nueve segundos, WhatsApp de dos minutos y cincuenta y nueve segundos, Twitter acepta textos de 280 caracteres, entre otras limitaciones que moldean y restringen los procesos narrativos, cognitivos y reflexivos.

Por último, la pluralidad, es decir, la variedad de expresiones, de aspectos, de tendencias o de características, por definición es lo opuesto a lo estandarizado, a lo único, a lo concentrado. El fraccionamiento de contenidos, su diseminación y dispersión a través múltiples plataformas y canales siguen siendo controladas por un mismo centro tecnocrático. La concentración no sólo nos conduce a la polarización y el reduccionismo en las narraciones, sino que nos somete a una hiper-mediación cada vez más dependiente de reglas tecnológicas que se erigen como una frontera infranqueable para un potencial paradigma alternativo. 


\section{SUMARIO FINAL}

A lo largo del texto hemos expuesto cómo la actual convergencia y concentración en info-comunicaciones opera sobre toda la cadena productiva y de servicios de medios, y para ellos hemos tomado como ejemplo en el caso argentino la fusión entre Telecom y Cablevisión. En este sentido, la paulatina coexistencia y superposición tecnológica de los canales de distribución tendría cuatro consecuencias inmediatas en la producción y distribución de productos y servicios de los medios. En primer lugar, efectos progresivos en la concentración de su propiedad en pocas manos. En segundo término, la concentración de la propiedad en pocas manos redunda en una posición de privilegio que permite imponer las reglas del juego al resto de los actores y captara sí una porción cada vez más amplia de ingresos. El tercer derivado de la convergencia y concentración info-comunicacional es la centralización geográfica en la generación de contenidos, afectando la pluralidad y profundizando las asimetrías culturales, políticas, económicas y sociales existentes en nuestro país. Y, en cuarto lugar, la acción multi-media y multi-plataforma operan una transformación sobre las representaciones, las narrativas y cadenas de sentido que adoptan, por ejemplo, los discursos periodísticos. En este sentido, el objetivo central no sería informar sino controlar la agenda de debates cuyo orden productivo busca, principalmente, construir credibilidad y respeto alrededor de una autoridad legitimada.

Asimismo, la tendencia tecnológica global de los medios de comunicación se inclina a desmasificar sus redes y dispositivos de contacto para crear segmentos diferenciados de públicos cada vez más identificados y discriminados en cuanto a prácticas, preferencias y consumos, pues su interés principal estaría enfocado en acceder a la vida íntima de los sujetos mediante canales y plataformas cada vez más personalizadas, en virtud de conseguir la mayor cuota de atención posible para saturarlos de información y estímulos específicos. El fraccionamiento de contenidos, su diseminación y dispersión a través múltiples plataformas y canales, no sólo nos conduce a un reduccionismo y una diversificación en las narraciones, sino que nos somete a una hipermediación dependiente de reglas tecnológicas cuyos márgenes cada vez más restringidos.

Situados en un paradigma caracterizado por el conocimiento, la información y las nuevas tecnologías, los medios con frecuencia logran una penetración comunicacional y un dominio en la construcción de las representaciones que presenta sus enfoques como una opción racional y de interés universal. Sin embargo, no se trata de intereses universales sino de intereses particulares que benefician a pequeñas minorías tecnocráticas altamente concentradas que han logrado perpetuarse en estructuras de poder elitistas heredadas del pasado. La orientación y el ordenamiento actual de las tecnologías no es algo dado e inmutable, pues la tecnología dispone de potencialidades que han sido cooptadas por un orden que controla y restringe la participación en su diseño y desarrollo.

Como líneas de desarrollo, reflexión y análisis futuro, surgen de este acercamiento al imbricado vínculo entre medios de comunicación y nuevas tecnologías, algunos pendientes. Por ejemplo, integrar un análisis que articule el universo de la producción de los medios y sus tecnologías con los estudios de recepción y la apropiación transformadora de las audiencias, tomando en cuenta los avances que se registran en la materia, cuyo enfoque se centra en los sujetos o usuarios como actores activos de una trama cultural simbólica, tanto a nivel de estímulos y contenidos como en los usos y prácticas respecto de la tecnología y los artefactos. En este sentido, si bien supera los objetivos del texto, advertimos una preocupación creciente y un progresivo proceso de transformación cultural basado en prácticas más participativas por parte de los usuarios, como resultado de un escenario más abierto, colaborativo y transversal en cuanto a las interacciones entre ciencia y sociedad, prácticas y consumos, recepción e investigación; como es el caso, por ejemplo, de proyectos como Ciencia Ciudadana (Citizen Science) que busca aplicar una serie de novedosos modelos de participación pública tendientes a recursos tecnológicos con valores pedagógicos, de creación colectiva, de protección del medio-ambiental, entre otros. 


\section{REFERENCIAS}

Althusser, L. (1999). La filosofía como arma de la revolución. Ciudad de México, México: Siglo XXI.

Aronson, E. (2000). El animal social. Madrid, España: Alianza.

Bernays, E. (1995). Cristalizando la opinión pública. Barcelona, España: Gestión.

Brown, J. A. C. (1991). Técnicas de persuasión. Madrid, España: Alianza.

Chomsky, N. (2007). El control de los medios de comunicación. Recuperado de https://www.voltairenet.org/ article145977.html

Elías, N. (1994). Conocimiento y poder. Madrid, España: Ediciones de la Piqueta.

Feenberg, A. (2005). Teoría crítica de la tecnología. Revista CTS, 2(5), 109-123.

Freud, S. (2010). La psicología de las masas y análisis del yo. Barcelona, España: Alianza.

Foucault, M. (2014).Vigilary Castigar. Nacimiento de la prisión. Ciudad Autónoma de Buenos Aires, Argentina: Siglo XXI.

González Casanova, P. (2004). Las nuevas ciencias y las humanidades. De la academia a la política. Barcelona, España: Anthropos.

Jenkins, H. (2008). Convergence culture. La cultura de la convergencia de los medios de comunicación. Barcelona, España: Paidós.

Lacan, J. (2013). Escritos 1. Barcelona, España: Biblioteca Nueva.

Lacunza, S. (2016) Pensar el periodismo. La crisis de la profesión y los medios analizada desde las redacciones. Ciudad Autónoma de Buenos Aires, Argentina: Ediciones B.

Marcuse, H. (1998). Technology, War and Fascism. Canada: Routledge.

Marx, C. (1969). El Capital. Ciudad de México, México: Siglo XXI.

Mastrini, G.; Becerra, M. (2006). Globalización, mercado e industrias culturales: ¿̇resistencia o simulacro? Trabajo presentado en el XII Encuentro Latinoamericano de Facultades de Comunicación Social. PUJ-Bogotá.

McChesney, R. W.; Nichols, J. (2002). Our Media, Not Theirs: the Democratic Struggle Against Corporate Media. New York, United States: Seven Stories.

McLuhan, M. (1964). El medio es el mensaje. Reino Unido: Penguin Books.

Ponza, P. (2018). Tesis, antítesis y opuestos complementarios en la prensa gráfica argentina. Las formaciones discursivas de La Nación y Página 12 frente a las políticas kirchneristas. Revista E-Latina. Revista de Estudios Latinoamericanos, 17(65), 62-81. https://publicaciones.sociales.uba.ar/index.php/elatina/article/view/3073/p df_117

Sosa Plata, G. (2016). Concentración de medios de comunicación, poder y nuevas legislaciones en América Latina. El Cotidiano, (195), 17-30. Recuperado de http://www.redalyc.org/articulo.oa?id=32543454003

Tilly, C. (2005). Los movimientos sociales entran en el siglo veintiuno. Política y Sociedad, 42(2), 11-35.

Tofler, A. (1979). La Tercera Ola. Maryland, Estados Unidos: Bantam Books.

Van Dijk, T. (2012). Discurso y contexto. Una aproximación cognitiva. Barcelona, España: Gedisa.

\section{Prensa de ACtualidad}

Becerra, M. (2019). Concentración mediática, un símbolo de la era Macri. Letra P. https://www.letrap.com.ar/nota/ 2019-4-15-16-53-0-la-concentracion-mediatica-un-simbolo-de-la-era-macri consultado el 22/04/2019.

Crettaz, J. (2017). La fibra óptica de Arsat tiene $22.500 \mathrm{~km}$ operativos y conecta 207 localidades. La Nación: https://www.lanacion.com.ar/tecnologia/la-fibra-optica-de-arsat-tiene-22500-km-operativos-y-conec ta-207-localidades-nid2012015 consultado el 7 de junio de 2019. 
Ensinck, M.G. (2019). Telecom será la tercera empresa en facturación de la Argentina. El Cronista: http://www.cronista.com/negocios/El-Gobierno-aprobo-la-fusion-Cablevision-Telecom-sera-la-terc era-empresa-en-facturacion-de-la-Argentina-20180629-0075.html consultada el 7 de junio de 2019.

Fernández, B. P. (2018). El Gobierno aprobó la fusión entre Cablevisión y Telecom. La Nación. Recuperado de https ://www.lanacion.com.ar/economia/el-gobierno-aprobo-la-fusion-entre-cablevision-y-telecom-nid2148955

Postolski, G., Santucho A. y Rodríguez D. (2003). IADE / REALIDAD ECONOMICA Revista Digital No1. http:/ /www.iade.org.ar/noticias/concentracion-de-los-medios-de-comunicacion-en-argentina-crisis-y-dependencia

Valli, P. (2018). Aprobaron la fusión de Telecom-Cablevisión y le imponen condiciones. Perfil. Recuperado de https://www.perfil.com/noticias/economia/aprobaron-la-fusion-de-telecom-cablevision-y-le-imponen-con diciones.phtml

Vásquez, C. (23 de marzo de 2017). La prensa y la dictadura: una estrecha relación bajo la lupa. La Izquierda a diario. Recuperado de https://www.laizquierdadiario.com/La-prensa-y-la-dictadura-una-estrecha-relacion-bajo-la-lup a

\section{Documentos}

Boletín Oficial de la República Argentina. ENACOM: Resolución 5644-E/2017 del 21/12/2017. Expediente EX-2017-20912532-APN-AMEYS\#. Ley N²7.078, Ley N²6.522, y Decreto No 267.

\section{BY-NC-SA}

